\title{
HIGHER ORDER COMPOSITIONS IN THE ADAMS SPECTRAL SEQUENCE
}

\author{
BY A. LAWRENCE
}

Communicated by P. Emery Thomas, December 3, 1969

Following is a summary of results relating to the convergence of Massey products in the Adams spectral sequence to Toda brackets in stable homotopy. These results should give much useful information on the classical structure problems; detecting infinite cycles, computing differentials and describing the extensions in $E_{\infty}$.

Forthcoming papers will treat Proposition II and Theorems III, IV, and V in full. R. M. F. Moss [6] has already proven Theorem III in the case of secondary compositions.

1. The Adams spectral sequence. We presuppose an appropriate stable category $S_{*}$ such as Boardman's C-W spectra [2]. Let $X, Y$ be objects of $S_{*}$. We denote Hom $s_{*}(X, Y)$ by $[X, Y]_{*}$. As in Adams [1], we may construct a spectral sequence $\left\{E_{r}^{* *}(X, Y)\right\}$ for which

$$
E_{2}^{* *}(X, Y)=\operatorname{Ext}_{A(2)}^{* *}\left(H^{*}\left(Y ; Z_{2}\right), H^{*}\left(X ; Z_{2}\right)\right)
$$

and $E_{\infty}^{* *}(X, Y)$ is induced by a filtration on the 2-primary part of $[X, Y]_{*}$.

The main technical results which enable us to obtain convergence of Massey products to Toda brackets are Propositions I and II below.

Proposition I (R. M. F. Moss). Let $X, Y, Z, W$ be objects of $\mathcal{S}_{*}$. For $2 \leqq r$ there are associative pairings

$$
E_{r}^{e, t}(Y, X) \otimes E_{r}^{a^{\prime}, t^{\prime}}(W, Y) \rightarrow E_{r}^{s+s^{\prime}, t+t^{\prime}}(W, X)
$$

and these pairings have the following properties:

(i) Let $\alpha \in E_{r}^{s, t}(Y, X), \quad \beta \in E_{r}^{s^{\prime}, t^{\prime}}(W, Y), \quad d_{r}^{\prime \prime}(\alpha \beta)=d_{r}(a) \beta+a d_{r}^{\prime}(\beta)$ where $d_{r}, d_{r}^{\prime}, d_{r}^{\prime \prime}$ are the differentials in the appropriate Adams spectral sequences.

(ii) The isomorphisms $E_{r+1} \cong H\left(E_{r}\right)$ commute with the pairings.

(iii) The composition pairings $[Y, X]_{p} \otimes[W, Y]_{q} \rightarrow[W, X]_{p+q}$ preserve filtration; on passing to quotients and using the isomorphisms of (ii) the composition pairing of the $E_{\infty}$ terms is obtained.

AMS Subject Classifications. Primary 5545, 5552.

Key Words and Phrases. Adams spectral sequence, Massey product, Toda bracket, splitting of Adams spectral sequence, convergence of Massey products in the Adams spectral sequence, generalized Leibnitz formulae. 
Proposition II. Suppose $X \stackrel{\jmath}{\rightarrow} Y \rightarrow C_{f}$ is a mapping cone sequence and $f$ projects to an element of $E_{\infty}^{s, *}(X, Y)$ in the Adams spectral sequence for some $s \geqq 2$. Then

$$
E_{r}^{* *}\left(C_{f}, Z\right) \cong E_{r}^{* *}(X, Z) \oplus E_{r}^{* *}(Y, Z)
$$

and

$$
E_{r}^{* *}\left(Z, C_{f}\right) \cong E_{r}^{* *}(Z, X) \oplus E_{r}^{* *}(Z, Y)
$$

for $r \leqq s$. Further, $d_{r}$ also splits for $r<s . d_{\text {s }}$ under case (a) has matrix

$$
\left(\begin{array}{ll}
d_{s} & 0 \\
f & d_{s}
\end{array}\right)
$$

and $d_{\mathrm{s}}$ under case (b) has matrix

$$
\left(\begin{array}{ll}
d_{s} & f \\
0 & d_{s}
\end{array}\right)
$$

2. Higher composition products. The definitions used in the construction of Toda brackets are those of Gershenson [3]; those used in the construction of Massey products are due to May [4].

Let $h^{i} \in\left[X^{i}, X^{i+1}\right]_{*}, i=1, \cdots, n$.

Definition. The Toda bracket $\left\langle h^{1}, \cdots, h^{n}\right\rangle$ exists if the following two conditions are satisfied.

(i) There is a coherent series of resolutions for $j=1, \cdots, n-1$

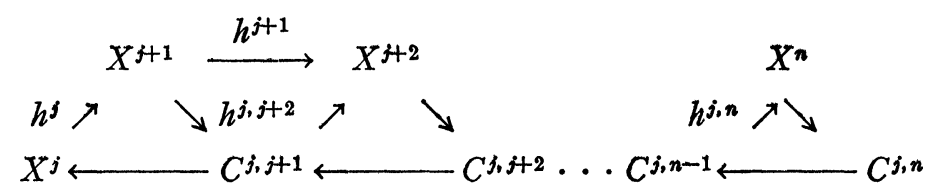

(The upper triangles commute, the lower are exact.) Such a series is called a $C$ presentation for $X \stackrel{h^{1}}{\rightarrow} X^{2} \rightarrow \cdots \stackrel{h^{n}}{\rightarrow} X^{n+1}$.

(ii) There are maps $h^{2, n}, \tilde{h}$ so that in the following diagram the upper triangle commutes, the lower is exact:

$$
\begin{aligned}
& X^{n} \stackrel{h^{n}}{\rightarrow} X^{n+1} \\
& \downarrow \quad \nearrow h^{2, n} \\
& C^{2, n} \\
& \downarrow \nwarrow \hbar \\
& C^{1, n} \rightarrow X
\end{aligned}
$$


Providing (i), (ii) are satisfied we define $\left\langle h^{1}, \cdots, h^{n}\right\rangle$ to be the set of $h^{2, n} \circ \tilde{h}$ obtained in (ii). Suppose we are given $v^{i} \in E_{\tau}^{* *}\left(X^{i}, X^{i+1}\right)$, $i=1, \cdots, n, r \geqq 2$.

Definition. We say the Massey product $\left\langle v^{1}, \cdots, v^{n}\right\rangle$ exists if we can find a collection of elements $a^{i j}$ (where $a^{i j} \in E_{r-1}^{* *}\left(X^{i}, X^{j}\right)$ ) so that the following conditions are satisfied:

(i) class $\left[a^{i, i+1}\right]=v^{i}, i=1, \cdots, n$,

(ii) $d a^{i j}=\sum_{k=t+1}^{j-1} a^{i k} a^{k j}, 1<(j-1)<n$.

Such a collection of elements $a^{i j}$ is said to be a defining system for $\left\langle v^{1}, \cdots, v^{n}\right\rangle$. We have that $\tilde{a}^{1, n+1}=\sum_{k=2}^{n} a^{1, k} a^{k, n+1}$ is a cycle and by definition $\left\langle v^{1}, \cdots, v^{n}\right\rangle$ is the set of all homology classes $\left[\tilde{a}^{1, n+1}\right]$ obtained via a defining system. Note that the bidegree of $a^{i, j}$ does not depend on the choice of defining system.

3. The convergence results. We fix $r \geqq 2$.

Theorem III. Let $v^{i} \in E_{r}^{* *}\left(X^{i}, X^{i+1}\right), i=1, \cdots, n$, be an infinite cycle. Assume that the Massey product $\left\langle v^{1}, \cdots, v^{n}\right\rangle$ is defined. Further, suppose that $v^{i} \Rightarrow h^{i}, h^{i} \in\left[X^{i}, X^{i+1}\right]$ and the Toda bracket $\left\langle h^{1}, \cdots, h^{n}\right\rangle$ is defined. If we are given the following two conditions:

(i) the Toda bracket $\left\langle h^{1}, \cdots, h^{n}\right\rangle$ is strictly defined, i.e., $\left\langle h^{i}, h^{i+1}\right.$, $\left.\cdots, h^{j}\right\rangle=\{0\}$ if $i>1$ or $j<n$;

(ii) whenever an element of a defining system for $\left\langle v^{1}, \cdots, v^{n}\right\rangle$ occurs in $E_{r-1}^{p, q}\left(X^{i}, X^{j}\right)$ then

$$
E_{r+u+w}^{p-w, q-w}\left(X^{i}, X^{j}\right) \subset E_{r+u+w, \infty}^{p-w, q-w} \quad \text { for } u \geqq 1, \quad w \geqq 0,
$$

then there is $v \in\left\langle v^{1}, \cdots, v^{n}\right\rangle$ and $h \in\left\langle h^{1}, \cdots, h^{n}\right\rangle$ so that $v \Rightarrow h$.

Theorem IV (LeIBNitz Formula). Let $\left\langle v^{1}, \cdots, v^{n}\right\rangle$ be a Massey product, where $v^{i} \in E_{\uparrow}^{* *}\left(X^{i}, X^{i+1}\right)$ and $d_{t} v^{i}=0, r \leqq t<s$. Assume that whenever $a$ term in a defining system for $\left\langle v^{1}, \ldots, v^{n}\right\rangle$ occurs in $E_{r-1}^{p, q}\left(X^{i}, X^{j}\right)$ then

$$
E_{t}^{p+t, q+t-1}\left(X^{i}, X^{j}\right)=0=E_{r+s-t}^{p+t, q+t-1}\left(X^{i}, X^{j}\right)
$$

where $s$ is fixed, $r<t<s$.

Then for $v \in\left\langle v^{1}, \cdots, v^{n}\right\rangle$ we have $d_{t} v=0, t<s$, and

$$
\left(d_{s} v, v\right) \in\left\langle\left(d_{s} v^{1}, v^{1}\right),\left(\begin{array}{cc}
v^{2} & 0 \\
d_{s} v^{2}, & v^{2}
\end{array}\right), \cdots,\left(\begin{array}{cc}
v^{n} & 0 \\
d_{s} v^{n}, & v^{n}
\end{array}\right)\right\rangle .
$$

We may generalize the Leibnitz formula in the following:

Theorem V. Let $\left\langle v^{1}, \cdots, v^{n}\right\rangle$ be defined in $E_{r}^{* *}\left(X^{1}, X^{n}\right)$. Let $l, s$ be fixed numbers, $s>r, l<n$. Assume $v^{i} \Rightarrow h^{i} \in\left[X^{i}, X^{i+1}\right]$ and $\left\langle h^{k}, \cdots\right.$, 
$\left.h^{k+l}\right\rangle$ is strictly defined for $1 \leqq k<k+l \leqq n$. Assume that whenever an element of a defining system for $\left\langle v^{1}, \cdots, v^{n}\right\rangle$ is in $E_{r-1}^{p, q}\left(X^{i}, X^{\prime}\right)$

(i) if $j-i \leqq l, u \leqq 0, E_{r+u+1}^{p+u, q+u-1}\left(X^{\natural}, X^{j}\right) \subset E_{r+u+1, \infty}$,

(ii) if $l<j-1<n, r<t<s, E_{t}^{p+t, q+t-1}\left(X^{i}, X^{j}\right)=0$,

(iii) if $l+1<j-i, r<t<s, E_{r+s-t}^{p+t, q+t-1}\left(X^{i}, X^{j}\right)=0$.

Then there is $v \in\left\langle v^{1}, \cdots, v^{n}\right\rangle$ such that $d_{t} v=0, t<s$. Further there is $b^{i} \in E_{r}^{* *}\left(X^{i}, X^{i+l+1}\right)$ such that $b^{i} \Rightarrow \tilde{h}^{i} \in\left\langle h^{i}, \cdots, h^{i+l}\right\rangle$. The $b^{i \prime} \mathrm{s}$ and $v$ are related through the fact that $\left\langle c^{1}, \cdots, c^{n-l}\right\rangle$ is defined in $E_{r}^{* *}\left(X^{1}, X^{n+1}\right)$ where

$$
\begin{aligned}
c^{1} & =\left(b^{1}, v^{1}\right) \\
\dot{\cdot} & \\
\dot{c}^{i} & =\left(\begin{array}{ll}
v^{i+l} & 0 \\
b^{i} & v^{i}
\end{array}\right) \\
\dot{\cdot} & \\
c^{n-l} & =\left(\begin{array}{l}
v^{n} \\
b^{n-l}
\end{array}\right)
\end{aligned}
$$

and we may choose $v$ so that $d_{s} v \in\left\langle c^{1}, \cdots, c^{n}\right\rangle$.

The technique of proof for Theorems III, IV, and V consists of reducing the theorems to the special case where $v^{i} \in E_{r}^{r-1, r-1}\left(X^{i}, X^{i+1}\right)$. The splitting theorem and the conditions on the differentials are then applied inductively to construct a $C$-presentation for a Toda bracket. In Theorem III the Toda bracket is the one in the hypotheses, in Theorems IV and V the element $d_{8} v$ is in our Toda bracket.

As an example of the use of Proposition II the maps $h$ and $h^{2, n}$ required in Theorem III pull back to $\bigoplus_{k=2}^{n} a^{1, k}$ and $\bigoplus_{k=2}^{n} a^{k, n+1}$ respectively in $E_{r-1}^{* *}\left(X^{1}, C^{2, n}\right)$ and $E_{r-1}^{* *}\left(C^{2, n}, X^{n+1}\right)$, where $\left\{a^{i j}\right\}$ is a defining system for $v \in\left\langle v^{1}, \cdots, v^{n}\right\rangle$ and $h^{2, n} \circ \tilde{h} \in\left\langle h^{1}, \cdots, h^{n}\right\rangle$.

\section{BIBLIOGRAPHY}

1. J. F. Adams, Stable homotopy theory, Springer-Verlag, Berlin and New York, 1964. MR $32 \# 3061$.

2. J. M. Boardman, Stable homotopy theory, University of Warwick, Coventry, England (unpublished notes).

3. H. Gershenson, Higher composition products, J. Math. Kyoto Univ. 5 (1965), 137. MR 32 \#8341.

4. J. Peter May, Matric Massey products (unpublished notes).

5. R. M. F. Moss, On the composition pairing of Adams spectral sequences, Proc. London Math. Soc. (3) 18 (1968), 179-192. MR $36 \# 3360$.

6. - On Massey products in the Adams spectral sequence (to appear).

NoRThWESTERN UnIVERSITY, Evanston. IlLINoIs 60201 